\title{
Numerical Investigation of the Time Discretization Impact on the Accuracy of a Point Target Localization by UWB Radar
}

\author{
Ján Buša ${ }^{1, \star}$, Dušan Kocur ${ }^{1, \star \star}$, and Mária Švecováa ${ }^{1, \star \star \star}$ \\ ${ }^{1}$ Faculty of Electrical Engineering and Informatics, Technical University of Košice, Košice, Slovakia
}

\begin{abstract}
UWB radar technologies enable localization of moving persons (targets) situated behind nonmetallic obstacles. Under exact knowledge of the propagation times, from the transmitting to the receiving antennas, of the radar emitted electromagnetic wave (time of arrival, TOA), a highly accurate target localization can be achieved. Since TOA estimates only are available, their use for target localization may result in a sizeable target localization error. In this paper we study the influence of TOA quantization on the point target localization accuracy using numerical simulation methods.
\end{abstract}

\section{Introduction}

In the last years, great efforts have been made to develop methods for the localization of human beings based on monitoring their respiratory motion $[1,5]$. For that purpose, ultra-wideband (UWB) sensors (radars) operating in the frequency band DC-5 GHz can be used with advantage. Electromagnetic waves occupying such frequency band can penetrate most nonmetallic materials with a small enough attenuation, and hence persons located behind such obstacles can be detected. On the other hand, due to the employment of the ultra-wide bandwidth, UWB sensors can provide fine range resolution (in order of centimetres), and hence a high accuracy of the target localization as well [2].

Deep analyses of the problem of the person localization by UWB sensor has shown that there are many factors affecting the accuracy of person localization. The accuracy of the so-called timeof-arrival (TOA) estimation (time necessary for electromagnetic wave emitted by the radar to travel from transmitting to receiving antennas) and the UWB radar antenna layout belongs among them. In this paper, the impact of TOA quantization (TOA estimation problem) and the geometry of the radar antennas (defining the radar antenna layout) on the accuracy of the localization of a point target behind a wall will be studied using numerical simulation methods.

\section{TOA based localization of a static point target behind a wall}

Let us consider a measurement equipment consisting of one transmitting antenna $T$ placed at the origin $(0,0,0)$ of the Cartesian coordinate system, and 4 receiving antennas $R_{i}, i=1,2,3,4$, placed

\footnotetext{
^e-mail: jan.busa@tuke.sk

$\star \star$ e-mail: dusan.kocur@tuke.sk

$\star \star \star$ e-mail: maria.svecova@tuke.sk
} 

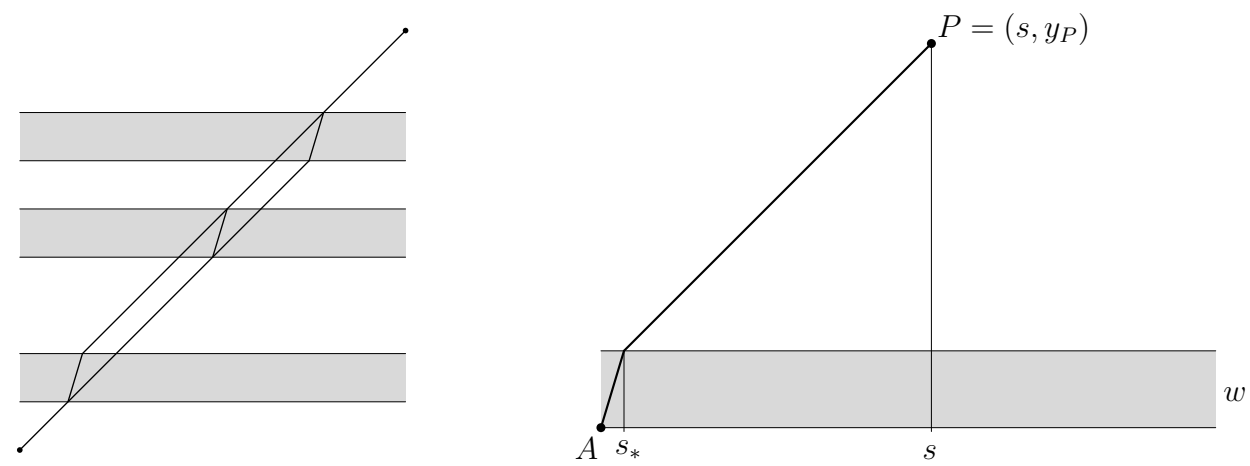

Figure 1. The wave propagation time does not depend on the wall position (left). The beam trajectory plane containing a point target $P$ and an antenna $A$ (right).

at the distance $d$ from the transmitting antenna at the points

$$
R_{1}=(d, 0,0), \quad R_{2}=(-d, 0,0), \quad R_{3}=(0,0, d), \quad R_{4}=(0,0,-d) .
$$

A concrete wall of width $w$ is assumed to be parallel to the plane $y=0\left(O_{x z}\right)$. The speed of light in the air, resp. within the wall we denote $c_{a}$, resp. $c_{w}$. Further let us suppose that a point target is at a point $P=\left(x_{P}, y_{P}, z_{P}\right)$.

An electromagnetic wave propagates from the transmitting antenna $T$ through the wall and air reaching the point target $P$, and after the reflection it propagates through the wall and air to the receivers $R_{i}, i=1, \ldots, n$ (in this paper we consider case $n=4$, see (1)). The time of the electromagnetic wave propagation from a transmitting to receiving antenna is denoted as the time of arrival (TOA). For these times, the notation such as $\mathrm{TOA}_{i}$ or $\operatorname{TOA}(P)_{i}, i=1, \ldots, n$, will be used throughout this paper.

An algorithm for the localization of a point target behind a wall based on the information about TOA to the receivers has been presented in [3]. Under the assumption of 4 or more available exact TOA values it is possible to determine not only the target position, but also the wall width (or the material relative permittivity).

In [4] the results of through-floor localization of a person using an ultra-wide band (UWB) radar are presented. A motionless person has been lying on the base floor at the position $P=$ $(-0.77 ; 2.84 ; 0.35)[\mathrm{m}](5$ antennas were placed on the first floor, and coordinate axis $y$ was directed down). Note that the respiratory motion is usually the only visible form of a movement for a static motionless person. For the $\mathrm{TOA}_{i}$ estimation, a reduced WP-STAPELOC method was employed [5]. The estimated position was $P_{\text {est }}=(-0.91 ; 2.71 ; 0.61)[\mathrm{m}]$, getting the localization precision $32.265 \mathrm{~cm}$.

Using some sampling frequency of the measurement one gets only the TOA values $\overline{\mathrm{TOA}}_{i}$ rounded (at least) to the closest upper sample time values. From a point of view of the rounded TOA values a points set of equivalency $\mathcal{E}(P)=\left\{\tilde{P} \in \mathbb{R}^{3} \mid \overline{\operatorname{TOA}}(\tilde{P})_{i}=\overline{\mathrm{TOA}}(P)_{i}\right\}$ (equivalency domain) can be defined. The impact of the time quantization (discretization) on the target localization accuracy - the shape and size of an equivalency domain $\mathcal{E}(P)$ - is presented below.

\section{Time of arrival calculation}

The TOA calculation in our simulations was based on the Fermat principle of least time, which leads to the Snell-Descartes law of refraction describing the relationship between the angles of incidence and refraction, when referring to the waves passing through a boundary between two different isotropic 

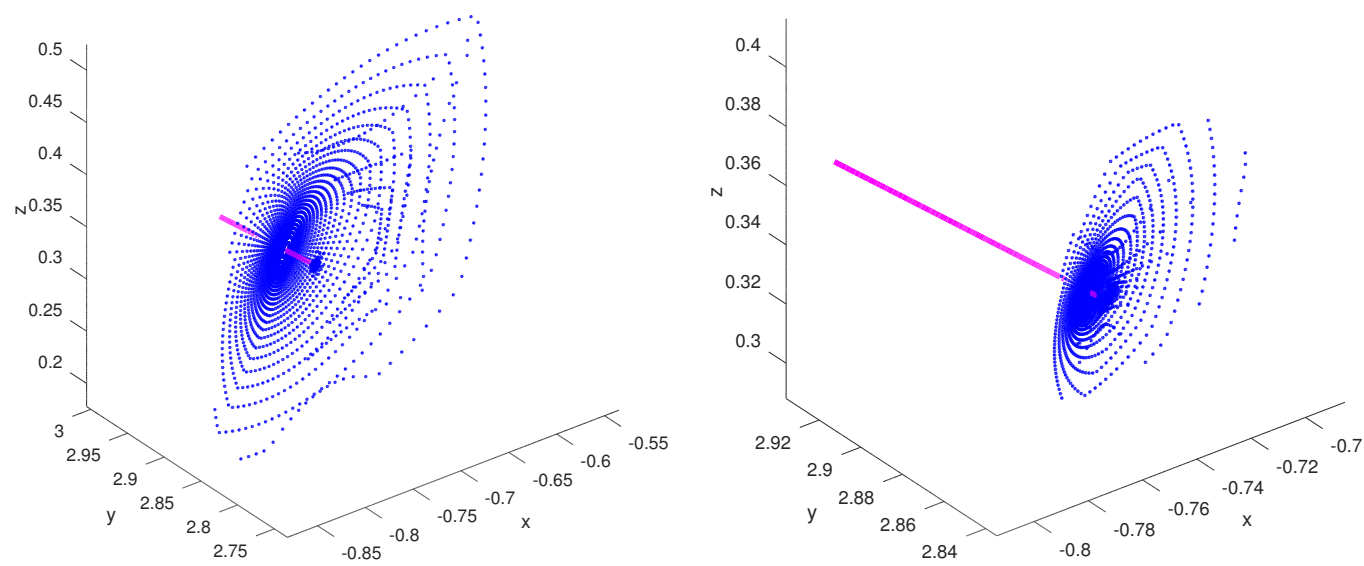

Figure 2. Equivalency domain $\mathcal{E}(P)$ boundary points for a point $P=(-0.77 ; 2.84 ; 0.35)[\mathrm{m}]$ for $3 \mathrm{GHz}$ (left) and $12 \mathrm{GHz}$ (right) sampling frequency (lengths of the arrows in radius vector $P$ direction are $10 \mathrm{~cm}$ )
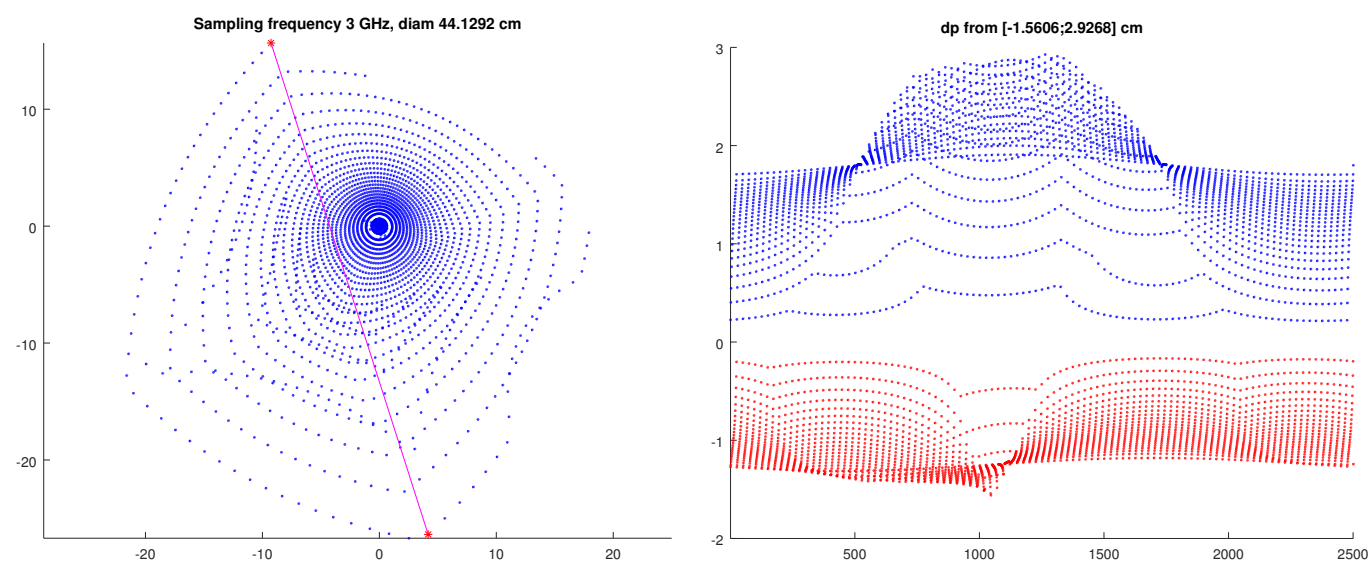

Figure 3. Projections of $\mathcal{E}(P)$ boundary points onto the plane orthogonal to the radius vector $P$ (left), distribution of the projections onto the line parallel to the radius vector $P$ (right) - sampling frequency $3 \mathrm{GHz}$

media, such as water, glass, or air. From the figure 1 left it is evident, that the wave propagation time does not depend on the wall position, so one side of the wall has been placed at the plane $y=0$. Applying the Fermat principle, minimizing the wave propagation time (see [3]), we get the following algebraic equation

$$
\left[c_{a}^{2}-c_{w}^{2}\right] \cdot s_{*}^{4}-2 s\left[c_{a}^{2}-c_{w}^{2}\right] \cdot s_{*}^{3}+\left[c_{a}^{2} y_{P}\left(y_{P}-2 w\right)+\left(c_{a}^{2}-c_{w}^{2}\right)\left(s^{2}+w^{2}\right)\right] \cdot s_{*}^{2}+2 c_{w}^{2} \cdot s \cdot w^{2} \cdot s_{*}-c_{w}^{2} \cdot w^{2} \cdot s^{2}=0
$$

for the determination of a value $s_{*}$, where $s=\sqrt{x_{P}^{2}+z_{P}^{2}}$ (Figure 1 right).

\section{Numerical results}

For selected points $P$ discretized values $\overline{\mathrm{TOA}}_{i}, i=1, \ldots, 4$ have been calculated. Relative permittivity 7.7 for reinforced concrete, receivers distance $d=0.8 \mathrm{~m}$, and the wall width $w=41 \mathrm{~cm}$ have been used. Afterwards for 5102 selected directions on a unit sphere using bisection method boundary points of the equivalency domain $\mathcal{E}(P)$ have been determined (Figure 2). 
The shape of the equivalency domain $\mathcal{E}(P)$ is flattened - its diameter for $3 \mathrm{GHz}$ sampling frequency size was $44.13 \mathrm{~cm}$ (the first row of Table 1), it was close to the diameter of the $\mathcal{E}(P)$ projection onto the plane orthogonal to the radius vector $P$ (Figure 3 left), and the deviations in the radius vector $P$ direction were from $d_{P_{\min }}=-0.938$ upto $d_{P_{\max }}=3.827 \mathrm{~cm}$ getting the equivalency domain width in the $P$ direction $4.765 \mathrm{~cm}$ (Table 1 and Figure 3 right).

Table 1. Precision dependence on the sampling frequency for a point $P=(-0.77 ; 2.84 ; 0.35)[\mathrm{m}]$

\begin{tabular}{cccccc} 
frequency $[\mathrm{GHz}]$ & $d_{\min }[\mathrm{cm}]$ & $d_{\max }[\mathrm{cm}]$ & $d_{P_{\min }}[\mathrm{cm}]$ & $d_{P_{\max }}[\mathrm{cm}]$ & diam $[\mathrm{cm}]$ \\
\hline 3 & 0.016 & 26.833 & -0.938 & 3.827 & 44.129 \\
6 & 0.016 & 17.542 & -0.938 & 1.277 & 22.747 \\
12 & 0.016 & 7.106 & -0.055 & 0.945 & 9.570
\end{tabular}

Table 2. Precision dependence on the sampling frequency for a point $P=(1 ; 10 ;-2)[\mathrm{m}]$

\begin{tabular}{cccccc} 
frequency $[\mathrm{GHz}]$ & $d_{\min }[\mathrm{cm}]$ & $d_{\max }[\mathrm{cm}]$ & $d_{P_{\min }}[\mathrm{cm}]$ & $d_{P_{\max }}[\mathrm{cm}]$ & $\operatorname{diam}[\mathrm{cm}]$ \\
\hline 3 & 0.414 & 93.829 & -3.777 & 1.253 & 142.245 \\
6 & 0.052 & 41.214 & -0.623 & 0.663 & 52.287 \\
12 & 0.052 & 28.612 & -0.568 & 0.609 & 36.641 \\
\hline $3^{*}$ & 0.710 & 60.564 & -2.881 & 1.777 & 78.774 \\
$6^{*}$ & 0.100 & 27.562 & -1.509 & 0.789 & 35.999 \\
$12^{*}$ & 0.100 & 11.794 & -0.770 & 0.306 & 18.473
\end{tabular}

* - receivers distance from the transmitting antenna $1.6 \mathrm{~m}$

Minimal, resp. maximal distances of boundary points from the point $P$ are denoted by $d_{\min }$, resp. $d_{\max }$ (Tables 1 and 2). The table 2 contains values for a point $P=(1 ; 10 ;-2)[\mathrm{m}]$.

\section{Conclusions}

Our investigation has shown that for a higher sampling frequency $12 \mathrm{GHz}$ the localization accuracy represented by the equivalency domain diameters is acceptable for practical using. For larger distances the accuracy is smaller. Larger receiving antennas distances lead to a higher localization precision. It would be interesting to consider equipment with 2 receivers, e.g., $R_{3}$ and $R_{4}$ shifted in the $x$ direction.

\section{Acknowledgments}

This work has been supported by the Scientific Grant Agency (VEGA) under the contract No. 1/0772/17 and by the Slovak Research and Development Agency under the contract No. APVV15-0692.

\section{References}

[1] E. Zaikov and J. Sachs, Ultra Wideband (InTech, Croatia, 2010) 323-346

[2] J. Sachs, Handbook of ultra-wideband short-range sensing: theory, sensors, applications (WileyVCH Verlag GmbH \& Co. KGaA., 2012) 843 pp.

[3] J. Buša, Numerical Methods and Applications (Springer, LNCS 8962, 2015) 254-261

[4] M. Švecová, D. Kocur, J. Demčák, J. Buša, and M. Gamcová, Electronics Letters (submitted 2017).

[5] D. Novák, M. Švecová, and D. Kocur, Microwave systems and applications (InTech, 2017) 399422 